\title{
Characteristics of confined exciton states in silicon quantum wires
}

\author{
D.V. Korbutyak, Yu.V. Kryuchenko, I.M. Kupchak, A.V. Sachenko \\ Institute of Semiconductor Physics, NASciences of Ukraine, 45 prospect Nauky, 03028 Kiev, Ukraine \\ Phone: +38(044)2656391,e-mail:div47@isp.kiev.ua
}

\begin{abstract}
We have studied theoretically the combined effect of quantum confinement and "dielectric enhancement" on the characteristics of the exciton ground state in quasi-1D silicon nanowires. Consideration has been made within effective mass and classical image force approximations. As a result, exciton binding energy, total energy of the exciton transition, radiative recombination time, intensity and internal quantum efficiency of the exciton photoluminescence $(\mathrm{PL})$ in quantum wires $(\mathrm{QW})$ have been obtained as functions of wire thickness, dielectric constants of adjacent materials, conduction and valence band-offsets. It was shown that even at room temperatures and moderate intensities of laser excitation the quantum efficiency of the exciton PL can achieve very high values (tens of \%) in the case of extremely thin QWs (with thickness $1 \div 3 \mathrm{~nm}$ ). Moreover, according to theory, the exciton recombination time and the quantum efficiency have to be oscillating functions of QW thickness in thickness range $1 \div 5 \mathrm{~nm}$ due to the indirect band-gap nature of silicon material.
\end{abstract}

Keywords: exciton, quantum confinement, binding energy, silicon nanotubes, photoluminescence, quantum efficiency.

Paper received 13.03.03; accepted for publication 16.06.03.

\section{Introduction}

In low-dimensional heterostructures, characteristics of electron, hole and exciton states are determined by quantum confinement conditions, such as nanostructure size and form, band-offsets at the heterointerfaces, dielectric properties of the adjacent media, etc. Different approximations are used considering such structures. One of the most popular is the effective mass method (it works well up to characteristic sizes of about 1-2 nm). Besides, in a number of works the approximation of infinitely large band-offsets (infinitely high barriers) is used. Within such approximation, it is possible to account for the "dielectric enhancement" effect (influence of interface polarization on the exciton binding energy) [1-3], but the approximation is too crude to give right values of quasiparticle energies in real structures with finite barriers. Contrary, in other models the effect of finite band-offsets is considered (see e.g.[4,5]), but only supposing no difference in dielectric constants of adjacent materials. This approximation allows avoiding difficulties connected with nonphysical divergence of classical electrostatic potential at heterointerfaces. In the present work, we have made calculations for silicon quantum wires taking into account both "dielectric enhancement" effect and finite band-offsets for conduction and valence bands at heterointerfaces. Classical electrostatic potentials in the near vicinity of heterointerfaces $(0.1-0.2 \mathrm{~nm})$ are replaced in our model by linear extrapolations from more distant regions at both sides of interface. Such extrapolations allow to operate with smooth and continuous total potentials (sum of the electrostatic and band-offset potentials in the whole actual region including interface. Due to practically complete compensation of the contributions from thin transition layers at opposite sides of interface into self-action energy shifts, the final results obtained in our approximations should be only slightly different from more complicated consideration (e.g., when spatial dispersion effects are taken into account).

\section{Wave functions and ground state energy of QW Wannier-Mott excitons}

In our model system, we consider isolated cylindrical quantum wire formed by material $l$ around QW and material 2 inside QW. Parameters of the system are as follows: isotropic effective electron masses $m_{e 1}$ and $m_{e 2}$, isotropic effective heavy hole masses $m_{h 1}$ and $m_{h 2}$, dielectric constants $\varepsilon_{l}$ and $\varepsilon_{2}$, conduction and valence band- 


\section{D.V. Korbutyak et al.: Characteristics of confined exciton states ...}

offsets at heterointerfaces $U_{e}$ and $U_{h}, \mathrm{QW}$ thickness $d$ (or radius $R=d / 2$ ). We consider crystal orientation that provides additional channel for radiative transitions in indirect band-gap materials like silicon. It is the geometry when four of six wave vectors $\boldsymbol{K}_{0 i}(i=1 \ldots 6)$ corresponding to the bottoms of the lowest $\mathrm{X}$-valleys in the conduction band are normal to the wire axis ( $\mathrm{z}$-axis in our notations). The wave function of the ground exciton state (for example, with $\boldsymbol{K}_{0 i}$ vector directed along $X$-axis) can be written as $\Phi_{K \|}=L^{-1 / 2} \exp \left(i K_{\|} Z\right) \exp \left(i K_{0 \mathrm{i}} x_{e}\right) \Psi\left(z, \rho_{e}, \rho_{h}\right)$, where $L$ is the QW length and $Z$ is the exciton center-ofmass coordinate along the wire axis. In this case $\Psi$-function of the internal motion of the electron-hole pair in the ground exciton state has to be a solution of the following Schrödinger equation:

$$
\begin{aligned}
& {\left[-\frac{\hbar^{2}}{2 m_{e}\left(\rho_{e}\right)} \Delta_{e}-\frac{\hbar^{2}}{2 m_{h}\left(\rho_{h}\right)} \Delta_{h}-\right.} \\
& -\frac{\hbar^{2}}{2 \mu\left(\rho_{e}, \rho_{h}\right)} \frac{d^{2}}{d z^{2}}+U_{s}\left(\rho_{e}\right)+U_{c}\left(\rho_{e}\right)+ \\
& \left.+U_{s}\left(\rho_{h}\right)+U_{v}\left(\rho_{h}\right)+U_{e h}\left(z, \rho_{e}, \rho_{h}\right)\right] \Psi\left(z, \rho_{e}, \rho_{h}\right)= \\
& =\left(E-E_{g}\right) \Psi\left(z, \rho_{e}, \rho_{h}\right),
\end{aligned}
$$

where $\rho_{i}=\sqrt{x_{i}^{2}+y_{i}^{2}}, \Delta_{i}=d^{2} / d \rho_{i}^{2}+\left(1 / \rho_{i}\right)\left(d / d \rho_{i}\right), i=$ $=e(h), z=z_{e}-z_{h}$. Potential energies $U_{s}\left(\rho_{e}\right)$ and $U_{s}\left(\rho_{h}\right)$ describe electron and hole self-action due to interfaces polarization, $U_{e h}\left(z, \rho_{e}, \rho_{h}\right)$ includes both direct Coulomb electron-hole interaction and indirect interaction via interface polarization. Bulk material characteristics of effective mass method $U_{c}\left(\rho_{e}\right)$ and $U_{v}\left(\rho_{h}\right)$ are the bottoms of conduction and valence bands as functions of distance from the wire axis. For material 2 inside QW $(\rho<R)$ $U_{c}=0$ and $U_{v}=0$ while for material 1 around QW $(\rho>R)$ $U_{c}=U_{e}$ and $U_{v}=U_{h}$. Energy $E_{g}$ is the bulk band-gap of a material 2. Using Green-function method [4] it is possible to obtain following explicit expressions for all potential energies in (1):

$$
\begin{aligned}
& U_{s}(\rho)=-\frac{e^{2}}{\pi} \frac{\varepsilon_{2}-\varepsilon_{1}}{\varepsilon_{1}} \sum_{m=0}^{\infty}\left(2-\delta_{m, 0}\right) \times \\
& \times \int_{0}^{\infty} \frac{I_{m}(\eta R) I_{m}^{\prime}(\eta R) \eta R}{\varepsilon_{1}+\left(\varepsilon_{2}-\varepsilon_{1}\right) K_{m}(\eta R) I_{m}^{\prime}(\eta R) \eta R} K_{m}^{2}(\eta \rho) d \eta \quad \text { if } r>R,
\end{aligned}
$$

$$
\begin{aligned}
& U_{s}(\rho)=-\frac{e^{2}}{\pi} \frac{\varepsilon_{2}-\varepsilon_{1}}{\varepsilon_{2}} \sum_{m=0}^{\infty}\left(2-\delta_{m, 0}\right) \times \\
& \times \int_{0}^{\infty} \frac{K_{m}(\eta R) K_{m}^{\prime}(\eta R) \eta R}{\varepsilon_{1}+\left(\varepsilon_{2}-\varepsilon_{1}\right) K_{m}(\eta R) I_{m}^{\prime}(\eta R) \eta R} I_{m}^{2}(\eta \rho) d \eta \quad \text { if } r<R .
\end{aligned}
$$

where $K_{m}$ and $I_{m}$ are the modified Bessel functions. Electron-hole interaction energy can be expressed as $U_{e h}\left(z, \rho_{e}, \rho_{h}\right)=\int_{0}^{\infty} \cos (\eta z) W_{e h}\left(\eta, \rho_{e}, \rho_{h}\right) d \eta$. For electron and hole outside $\mathrm{QW}$, when both $\rho_{e}$ and $\rho_{h}>R$,

$$
\begin{aligned}
& W_{e h}\left(\eta, \quad \rho_{e}, \rho_{h}\right)=-\frac{2 e^{2}}{\varepsilon_{1} \pi} \times \\
& \times\left[I_{0}\left(\eta \rho_{<}\right) K_{0}\left(\eta \rho_{>}\right)-\left(\varepsilon_{2}-\varepsilon_{1}\right) \times\right. \\
& \left.\times \frac{I_{0}(\eta R) I_{0}^{\prime}(\eta R) \eta R}{\varepsilon_{1}+\left(\varepsilon_{2}-\varepsilon_{1}\right) K_{0}(\eta R) I_{0}^{\prime}(\eta R) \eta R} K_{0}\left(\eta \rho_{e}\right) K_{0}\left(\eta \rho_{h}\right)\right] .
\end{aligned}
$$

For electron inside QW and hole outside QW or for inverse situation

$$
\begin{aligned}
& W_{e h}\left(\eta, \quad \rho_{e}, \rho_{h}\right)=-\frac{2 e^{2}}{\varepsilon \pi} I_{0}\left(\eta \rho_{<}\right) K_{0}\left(\eta \rho_{>}\right) \times \\
& \times\left[1-\left(\varepsilon_{2}-\varepsilon_{1}\right) \frac{K_{0}(\eta R) I_{0}^{\prime}(\eta R) \eta R-0.5}{\varepsilon_{1}+\left(\varepsilon_{2}-\varepsilon_{1}\right) K_{0}(\eta R) I_{0}^{\prime}(\eta R) \eta R}\right],
\end{aligned}
$$

where $\varepsilon=\left(\varepsilon_{1}+\varepsilon_{2}\right) / 2$. Finally, for both electron and hole inside QW, when $\rho_{e}$ and $\rho_{h}<R$,

$$
\begin{aligned}
& W_{e h}\left(\eta, \rho_{e}, \rho_{h}\right)=-\frac{2 e^{2}}{\varepsilon_{2} \pi} \times \\
& \times\left[I_{0}\left(\eta \rho_{<}\right) K_{0}\left(\eta \rho_{>}\right)-\left(\varepsilon_{2}-\varepsilon_{1}\right) \times\right. \\
& \left.\times \frac{K_{0}(\eta R) K_{0}^{\prime}(\eta R) \eta R}{\varepsilon_{1}+\left(\varepsilon_{2}-\varepsilon_{1}\right) K_{0}(\eta R) I_{0}^{\prime}(\eta R) \eta R} I_{0}\left(\eta \rho_{e}\right) I_{0}\left(\eta \rho_{h}\right)\right] .
\end{aligned}
$$

In expressions (4)-(6) $\rho_{>}=\rho_{e}$ and $\rho_{<}=\rho_{h}$ if $\rho_{e}<\rho_{h}$ or $\rho_{<}=\rho_{h}$ and $\rho_{>}=\rho_{e}$ if $\rho_{h}<\rho_{e}$. Taking into account the equality [6]

$\frac{1}{\left|\mathbf{r}_{e}-\mathbf{r}_{h}\right|}=\frac{2}{\pi} \sum_{m=0}^{\infty}\left(2-\delta_{m, 0}\right) \cos \left[m\left(\varphi_{e}-\varphi_{h}\right)\right] \times$

$\times \int_{0}^{\infty} \cos \left[\eta\left(z_{e}-z_{h}\right)\right] I_{0}\left(\eta \rho_{<}\right) K_{0}\left(\eta \rho_{>}\right) d \eta$

and transversal isotropy of the ground state wave function it is evident that first term in the right-hand side of Eqs. (4)-(6) is responsible for direct Coulomb interaction between electron and hole while second describes indirect interaction via interface polarization (image force potential).

We have calculated the exciton spectrum using wave function decomposition over eigenstates of a model quasi$1 \mathrm{D}$ system. Within this method wave functions of confined exciton states in QW are constructed from the relevant eigenstates of a model Hamiltonian for which transversal and longitudinal motions of carriers can be separated: 


\section{D.V. Korbutyak et al.: Characteristics of confined exciton states ...}

$$
\begin{aligned}
& \Psi\left(z, \rho_{e}, \rho_{h}\right)=\sum_{m n k l j} C_{m n, k l, j} F_{j}(z) \chi_{e_{m n}}\left(\rho_{e}\right) \times \\
& \times \chi_{h_{k l}}\left(\rho_{h}\right) \exp \left(i m \varphi_{e}\right) \exp \left(-i k \varphi_{h}\right) .
\end{aligned}
$$

In the above expression $C_{m n, k l, j}$ are the expansion coefficients, $n(l)$ are the radial quantum numbers of confined electron (hole) states, $m(k)$ are the azimuthal quantum numbers of these states, $j$ are the quantum numbers of bound electron-hole states in a model system with quasi$1 \mathrm{D}$ Coulomb interaction. The accuracy of this method is limited by a number of terms in the wave function decomposition. For the exciton ground state only terms with $m=0$ and $k=0$ in $(8)$ are important.

Wave functions $\chi_{i_{m n}}\left(\rho_{i}\right)$ for carrier transversal motion in rectangular potential gaps and the corresponding eigenenergies $E_{i_{m n}}$ can be easily found in a standard way. E.g. for the states with $m=0(k=0)$ the following eigenvalue problem has to be solved:

$$
\left[-\frac{\hbar^{2}}{2 m_{i 1}} \Delta_{i}+\tilde{U}_{i}\right] \chi_{i 1}\left(\rho_{i}\right)=E_{i} \chi_{i 1}\left(\rho_{i}\right) \text { for } \rho_{i}>R, \text { and }
$$

$$
-\frac{\hbar^{2}}{2 m_{i 2}} \Delta_{i} \chi_{i 2}\left(\rho_{i}\right)=E_{i} \chi_{i 2}\left(\rho_{i}\right) \text { for } \rho_{i}<R,
$$

with boundary conditions $\chi_{i 1}\left(R_{+}\right)=\chi_{i 2}\left(R_{-}\right)$and $\left(1 / m_{i 1}\right) \quad \chi_{i 1}^{\prime}\left(R_{+}\right)=\left(1 / m_{i 2}\right) \quad \chi_{i 2}^{\prime}\left(R_{-}\right)$. Potential energy $\tilde{U}_{i}$ in (9) is the renormalized barrier height, $\tilde{U}_{i}=$ $=U_{i}-U_{s}(0)$, where $U_{s}(0)$ is the energy shift at the cylinder axis due to carrier self-action via interface polarization:

$$
\begin{aligned}
& U_{s}(0)=-\frac{e^{2}}{\pi} \frac{\varepsilon_{2}-\varepsilon_{1}}{\varepsilon_{2}} \times \\
& \times \int_{0}^{\infty} \frac{K_{0}(\eta R) K_{0}^{\prime}(\eta R) \eta R}{\varepsilon_{1}+\left(\varepsilon_{2}-\varepsilon_{1}\right) K_{0}(\eta R) I_{0}^{\prime}(\eta R) \eta R} d \eta .
\end{aligned}
$$

Appropriate solutions are $\chi_{i 1}=a_{i 1} K_{0}\left(k_{i 1} \rho_{i}\right)$ and $\chi_{i 2}=a_{i 2} J_{0}\left(k_{i 2} \rho_{i}\right)$ with $k_{i 1}=\sqrt{2\left(\tilde{U}_{i}-E_{i}\right) m_{i 1}} / \hbar$ and $k_{i 2}=\sqrt{2 E_{i} m_{i 2}} / \hbar$. Here $E_{i}$ are the energies measured from the renormalized (by self-action energy shifts $U_{s}(0)$ ) conduction $(i=e)$ and valence $(i=h)$ band bottoms of bulk material 2 . They can be found from the following equation:

$$
\frac{m_{i 2}}{m_{i 1}} \frac{k_{i 1}}{k_{i 2}} \frac{J_{0}\left(k_{i 2} R\right)}{J_{1}\left(k_{i 2} R\right)} \frac{K_{1}\left(k_{i 1} R\right)}{K_{0}\left(k_{i 1} R\right)}=1 .
$$

Coefficients $a_{i 1}$ and $a_{i 2}$ can be determined from the boundary conditions and the wave function normalization condition $2 \pi \int_{0}^{\infty} \rho \chi_{i}^{2}(\rho) d \rho=1$, where $\chi_{i}=\chi_{i 1}$ if $\rho>R$ and $\chi_{i}=\chi_{i 2}$ if $\rho<R$.
As to the longitudinal (along the wire) relative electron-hole motion, it is convenient to use eigenfunctions of the following model 1D Schrödinger equation :

$$
-\left[\frac{\hbar^{2}}{2 \mu_{22}} \frac{d^{2}}{d z^{2}}-\tilde{W}_{C}(z)\right] F_{j}(z)=E_{j} F_{j}(z),
$$

where second term in square brackets $\tilde{W}_{C}=$ $=-e^{2} / \varepsilon_{2}(|z|+a)$ approximates direct Coulomb interaction $W_{c}=-e^{2} / \varepsilon_{2}\left|\mathbf{r}_{e}-\mathbf{r}_{h}\right|$ between electron and hole in a wire, $m_{22}$ is the reduced exciton mass in material $2, m_{22}=$ $=m_{e 2} m_{h 2} /\left(m_{e 2}+m_{h 2}\right)$. The non-zero positive parameter $a$ in $\tilde{W}_{C}$ term allows to operate with complete system of exciton states in $\mathrm{QW}$, including the exciton ground state [7]. If $a \rightarrow 0$, the binding energy of the ground state tends to infinity while corresponding wave function transforms into $\delta$-function. For this reason the approximation of Coulomb interaction by the term $-e^{2} / \varepsilon_{2}|z|$ leads to the loss of the lowest state (and, as a result, to incompleteness of the eigenfunction system); calculations made with such conventional 1D Coulomb interaction (see, e.g. [8]) cannot be satisfactory .

Final results should be independent of a specific value of the parameter $a$. However, it is convenient to operate with optimized value of this parameter for quick convergence of the results. If the lowest confined exciton state in $\mathrm{QW}$ is a subject of interest, then the value of the parameter $a$ should be calculated from the requirement for best convergence of the results just for this state. It is equivalent to the requirement of best approximation by $\tilde{W}_{C}$ term of the real Coulomb interaction averaged over carrier transversal motion in the ground state. Practically, it is sufficient to require a coincidence between $\tilde{W}_{C}$ value at $z=0$ and the analogous value of the averaged Coulomb interaction. For bound $e-h$ states the solutions of Eq. (12) can be expressed via the Whittaker functions [7], $F_{j}(z)=C_{j} W_{\alpha_{j}, 1 / 2}(\tilde{z})[z /|z|]^{p}$, where $C_{j}$ is the normalization constant, $p$ is the parity index ( $p=1$ for odd states and $p=2$ for even states $), \alpha_{j}=\left(-E_{j} / E_{B}\right)^{-1 / 2}, E_{B}=$ $e^{4} \mu_{22} /\left(2 \varepsilon_{2}^{2} \hbar^{2}\right), \tilde{z}=2(|z|+a) /\left(\alpha_{j} r_{B}\right), r_{B}=\hbar^{2} \varepsilon_{2} /\left(e^{2} \mu_{22}\right)$. From the continuity of the wave function $F$ and its flux at $z=0$ the dispersion equations $\mathrm{W}_{\alpha, 1 / 2}^{\prime}\left(2 a /\left(r_{B} \alpha\right)\right)=0$ for even and $\mathrm{W}_{\alpha, 1 / 2}\left(2 a /\left(r_{B} \alpha\right)\right)=0$ for odd states can be obtained. These dispersion equations determine quantum numbers $\alpha_{j}$ and corresponding eigenenergies $E_{j}$.

With the above basic system of eigenfunctions the algebraic equations for the decomposition coefficients $C_{m n, k l, j}$ take up the following form:

$$
\begin{aligned}
& \left(E-E_{g}-E_{e_{m n}}-E_{h_{k l}}-E_{j}\right) C_{m n, k l, j}- \\
& -\sum_{m^{\prime} n^{\prime} k^{\prime} l^{\prime} j^{\prime}} T\left(m, m^{\prime} ; n, n^{\prime} ; k, k^{\prime} ; l, l^{\prime} ; j, j^{\prime}\right) C_{m^{\prime} n^{\prime}, k^{\prime} l^{\prime}, j^{\prime}}=0,
\end{aligned}
$$

where $T(\ldots)$ are the matrix elements of the original Hamiltonian with real 3D Coulomb interactions in the considered basis of model wave functions. Energy spectrum $E_{N}(N=1,2, \ldots)$ of the exciton states in QW can be obtained by solving secular equation for this system; corresponding coefficients $C_{m n, k l, j}^{N}$ are determined by (13) and

$S Q O, 6(2), 2003$ 


\section{D.V. Korbutyak et al.: Characteristics of confined exciton states ...}

normalization equation for the wave-function $\Psi_{N}\left(z, \rho_{e}, \rho_{h}\right)$.

For a verification of the results obtained by the above described perturbation theory method (for shortness referred in the following as matrix method) we have calculated also the energy characteristics of the ground exciton state in QW by variational method. We have chosen the variational ground state wave function in the form of $\Psi\left(z, \rho_{e}, \rho_{h}\right)=F(z) \chi_{e}\left(\rho_{e}\right) \chi_{h}\left(\rho_{h}\right)$ with $F(z)=\alpha^{1 / 2} \times$ $\times \exp (-\alpha|z|)$, where $\alpha$ is the variational parameter, $\chi_{e}\left(\rho_{e}\right)$ and $\chi_{h}\left(\rho_{h}\right)$ are the wave functions of the lowest confined electron and hole states in a wire which can be found from (9). Eigenenergies $E_{i}$ of the confined states measured from the renormalized conduction $(i=e)$ and valence $(i=h)$ band edges of bulk material 2 can be found from the following equation:

$$
\frac{m_{i 2}}{m_{i 1}} \frac{k_{i 1}}{k_{i 2}} \frac{J_{0}\left(k_{i 2} R\right)}{J_{1}\left(k_{i 2} R\right)} \frac{K_{1}\left(k_{i 1} R\right)}{K_{0}\left(k_{i 1} R\right)}=1 .
$$

Despite strong peculiarity at $z=0$ in the first derivative of the variational wave function the asymptotic expression for the full energy is an analytical function of the parameter $\alpha, E(\alpha)=E_{g}+2 U_{s}(0)+E_{e}+E_{h}+E_{x}(\alpha)+$ $+U_{s e}+U_{s h}$. Here $E_{x}$ is the exciton binding energy, $U_{s e}$ and $U_{s h}$ are the contributions from electron and hole renormalized potentials, which include the coordinate dependent rests of image force potentials. For $E_{x}$ the following expression can be obtained:

$$
\begin{aligned}
& E_{x}(\alpha)=\frac{\hbar^{2} \alpha^{2}}{2}\left[\frac{K_{1}^{2}\left(k_{e 1} R\right)}{K_{0}^{2}\left(k_{e 1} R\right)}+\frac{J_{1}^{2}\left(k_{e 2} R\right)}{J_{0}^{2}\left(k_{e 2} R\right)}\right]^{-1} \times \\
& \times\left[\frac{K_{1}^{2}\left(k_{h 1} R\right)}{K_{0}^{2}\left(k_{h 1} R\right)}+\frac{J_{1}^{2}\left(k_{h 2} R\right)}{J_{0}^{2}\left(k_{h 2} R\right)}\right]^{-1} \times \\
& \times\left\{\frac{m_{e 2}+m_{h 2}}{m_{e 2} m_{h 2}}\left(\frac{J_{1}^{2}\left(k_{e 2} R\right)}{J_{0}^{2}\left(k_{e 2} R\right)}+1\right)\left(\frac{J_{1}^{2}\left(k_{h 2} R\right)}{J_{0}^{2}\left(k_{h 2} R\right)}+1\right)+\right. \\
& +\frac{m_{h 2}-m_{h 1}}{m_{h 2} m_{h 1}}\left(\frac{K_{1}^{2}\left(k_{h 1} R\right)}{K_{0}^{2}\left(k_{h 1} R\right)}-1\right)\left(\frac{J_{1}^{2}\left(k_{e 2} R\right)}{J_{0}^{2}\left(k_{e 2} R\right)}+1\right)+ \\
& +\frac{m_{e 2}-m_{e 1}}{m_{e 2} m_{e 1}}\left(\frac{K_{1}^{2}\left(k_{e 1} R\right)}{K_{0}^{2}\left(k_{e 1} R\right)}-1\right)\left(\frac{J_{1}^{2}\left(k_{h 2} R\right)}{J_{0}^{2}\left(k_{h 2} R\right)}+1\right)+ \\
& +\left[\frac{m_{h 2}-m_{h 1}}{m_{h 2} m_{h 1}}+\frac{m_{e 2}-m_{e 1}}{m_{e 2} m_{e 1}}\right]\left(\frac{K_{1}^{2}\left(k_{h 1} R\right)}{K_{0}^{2}\left(k_{h 1} R\right)}-1\right) \times \\
& \times\left(\frac{K_{1}^{2}\left(k_{e 1} R\right)}{K_{0}^{2}\left(k_{e 1} R\right)}-1\right)\left(\int_{0}^{\infty} \frac{8 \alpha^{2}}{\eta^{2}+4 \alpha^{2} G_{e h}(\eta) d \eta}\right.
\end{aligned}
$$

where $G_{e h}(\eta)=4 \pi^{2} \int_{0}^{\infty} \rho_{e} \chi_{e}^{2}\left(\rho_{e}\right) d \rho_{e} \int_{0}^{\infty} W_{e h}\left(\eta, \rho_{e}, \rho_{h}\right) \times$ $\times \rho_{h} \chi_{h}^{2}\left(\rho_{h}\right) d \rho_{h}$. Self-action energy shifts are given by the expression $U_{s i}=2 \pi \int_{0}^{\infty} \rho \chi_{i}^{2}(\rho) \tilde{U}_{s}(\rho) d \rho$, where $\tilde{U}_{s}(\rho)=$ $=U_{s}(\rho)-U_{s}(0)$ inside and $\tilde{U}_{s}(\rho)=U_{s}(\rho)$ outside the wire. The problem of nonphysical divergence of self-action potential energy $\tilde{U}_{s}(\rho)$ at $\rho \rightarrow R$ can be bypassed using linear $\tilde{U}_{s}(\rho)$ extrapolations in thin interface layer $(0.1 \div 0.2 \mathrm{~nm}$ at both sides of interface) from more distant regions. Transition layer boundaries can be found approximately from a requirement for potential smoothness. In the case of $\varepsilon_{2}>\varepsilon_{1}$ it transforms into requirement of equal $\tilde{U}_{s}(\rho)$ slopes at opposite boundaries of the transition layer and coinciding values of the extrapolated full one-particle potentials $U_{s}(\rho)+U_{c, v}(\rho)$, at the interface. For $\varepsilon_{2}<\varepsilon_{1}$ the transition layer boundaries can't be determined in that way. Nevertheless, in this case too, it is possible to use classical image force potentials up to $\sim 0.1$ $\mathrm{nm}$ vicinity of the interface and linearly extrapolated values of potentials in thin transition layer like in [9]. Calculations have shown that after explicit evolvement of $U_{s}(0)$ energy shifts (inside QW) the contribution into the total energy of remaining parts of self-action becomes small enough comparing to other terms even at large potential energies within transition layer. Physically, it is a result of practically complete compensation of remaining self-action contributions from opposite sides of the interface, so that the above extrapolations (which provide finite values of potential energies at the interface) seems to be justified. Infinitely large values of potential energies at the interface are in fact the result of the classical approach in which abrupt change in dielectric constant at the interface is assumed. This approximation becomes invalid for the carriers in transition layer in the near vicinity of the interface. To solve this problem more strongly and obtain realistic continuous finite electrostatic potentials in transition layer the effects of spatial dispersion should be taken into account. However, in this case consideration becomes much more complicated. Moreover, it is evident that such complicated consideration can give only small corrections to our results due to outlined compensation effect in the transition layer.

Fig. 1 illustrates the calculated potential gaps (thick solid curves) for electrons and holes in $\mathrm{Si}_{-} \mathrm{SiO}_{\mathrm{x}} \mathrm{QW}$ structure with account of self-action image force potentials in a particular case of $x=1.5$ and wire diameter $d=1.5 \mathrm{~nm}$. Traditional potential energy diagram for the QW structure with conduction and valence band-offsets at wire walls is shown by thin solid line, the bottoms of the lowest conduction and valence subbands by dashed lines, and squares of transversal motion wave functions in these subbands by dashed-dotted curves (the last in arbitrary units). It is evident from this figure that interface polarization shifts substantially bottoms of potential gaps and subbands in QW structures increasing e-h transition energy by the value of $\sim 2 U_{s}(0)$.

Dependencies of the exciton binding energies on the QW diameter $d$ are shown in Fig. 2. Calculations have been made for the parameters of a material inside QW 


\section{D.V. Korbutyak et al.: Characteristics of confined exciton states ...}

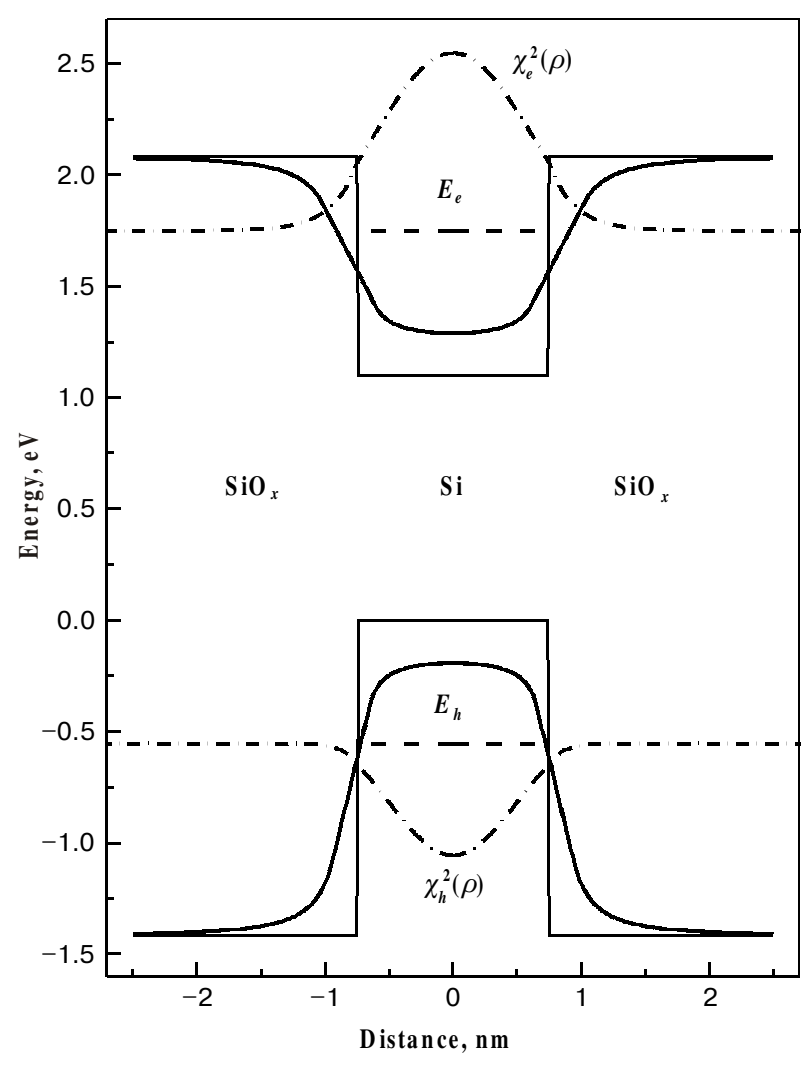

Fig. 1. Model of silicon quantum wire in $\mathrm{SiO}_{\mathrm{x}}$ matrix.

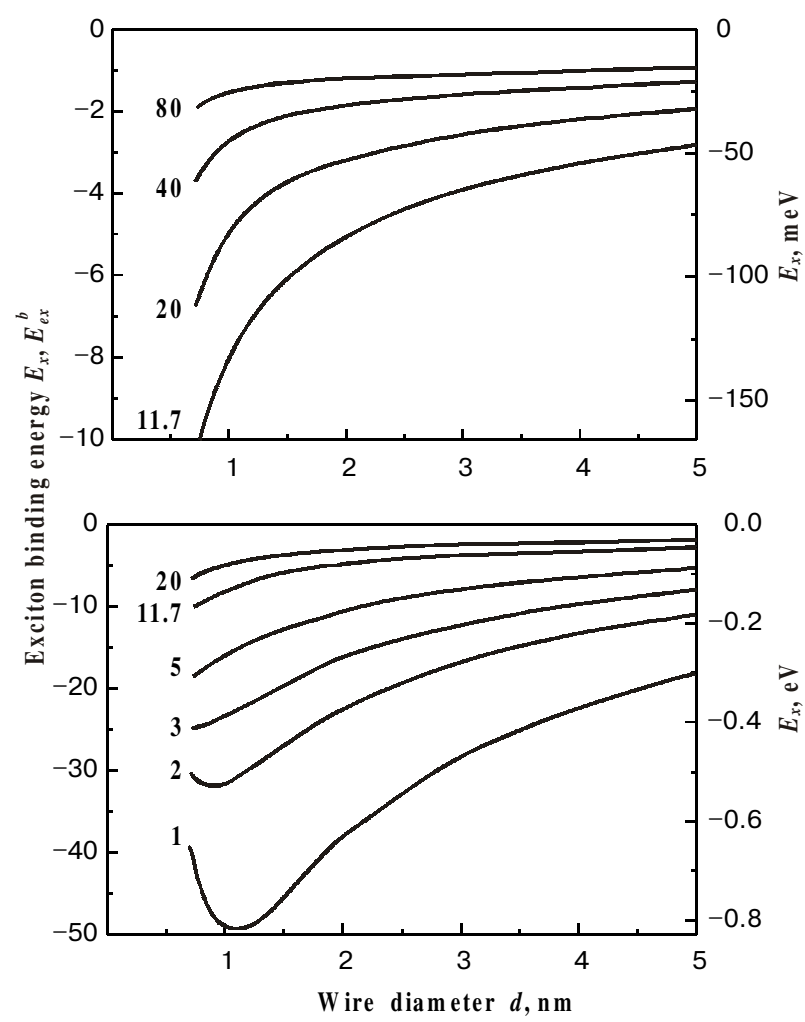

Fig. 2. Binding energies of the Wannier-Mott exciton in QW as functions of wire diameter. Numbers at curves are the corresponding dielectric constants $\varepsilon_{1}$ of a barrier material.. close to those of silicon (effective electron mass $m_{e 2}=0.25$ $m_{0}$, effective heavy hole mass $m_{h 2}=0.5 m_{0}$, dielectric constant $\left.\varepsilon_{2}=11.7\right)$. The bulk value of exciton binding energy in this material is $E_{e x}^{b}=16.6 \mathrm{meV}$. As to the barrier region, the parameters of $\mathrm{SiO}_{2}$ material have been used except effective dielectric constant $\varepsilon_{1}$. With this dielectric constant as a variable parameter we account effectively for more complex cases, when besides adjacent $\mathrm{SiO}_{2}$ layer at $\mathrm{QW}$ there are other components in a system (e.g., vacuum pores in porous silicon). This figure demonstrates substantial role of both confinement and dielectric enhancement effects which are responsible for very large (tenth of $\mathrm{eV}$ ) exciton binding energies in $\mathrm{QW}$ nanostructures.

To demonstrate the total effect of quantum confinement, finite barrier height and interface polarization on the exciton ground state characteristics, we have considered silicon $\mathrm{QW}$ in $\mathrm{SiO}_{x}$ matrix. In accordance with the data of $[10,11]$, the band gap of $\mathrm{SiO}_{x}$ material varies in a wide range from $\sim 1.7 \mathrm{eV}$ at low oxygen content to $\sim 8.9 \mathrm{eV}$ at $x \sim 2$. For $\mathrm{SiO}_{\mathrm{x}}$ material we have used linear interpolations $m_{e 1}=(0.25+0.125 x) m_{0}$ and $m_{h 1}=(0.5+0.25 x) m_{0}$ between values of effective masses at $x=0$ and $x=2$ [12]. Dielectric constant $\varepsilon_{1}$ of $\mathrm{SiO}_{x}$ material at different $x$ values has been determined numerically from the data presented in [11]; it changes from $\varepsilon_{1} \sim 2.1$ at $x=2$ to $\varepsilon_{1} \sim 11.7$ at $x=0$. The band-offsets in $\mathrm{Si}_{-}-\mathrm{SiO}_{2}$ structure are $3.2 \mathrm{eV}$ for the conduction band and $4.6 \mathrm{eV}$ for the valence band [12]. At arbitrary $x$ values band-offsets have been determined from the data for $\mathrm{SiO}_{\mathrm{x}}$ band gap $[10,11]$ suggesting that ratio $U_{e} / U_{h}=3.2 / 4.6$ is approximately valid at all actual $x$ values. Fig. 3 illustrates results for silicon quantum wires in $\mathrm{SiO}_{\mathrm{x}}$ material. Both variational and matrix methods have been used for a comparison. Noticeable decrease of variational values of exciton binding energy at small QW thicknesses is a consequence of increasing failure of the system description at such thicknesses by our simple variational function. At small thicknesses more complex variational function should be used. Accounting for finite barriers, at extremely small $d$ it must transform into wave function of bulk exciton.

Dependencies obtained by the perturbation (matrix) method are more realistic because their improper behavior manifests at smaller QW diameters than in variational method. With larger systems of model Hamiltonian eigenstates further improvement within matrix method is available. Despite discussed limitations, in actual range of QW widths the variational function and perturbation theory results are close to each other and can be considered as sufficiently reliable.

In Fig. 4 exciton and lowest $e-h$ recombination energies in $\mathrm{Si}-\mathrm{SiO}_{2} \mathrm{QW}$ are compared with the energies obtained assuming infinitely high barriers for electrons and holes. Thickness dependencies of the band gap between lowest conduction and valence subbands are shown by solid curves, while analogous dependencies for the exciton transition energies by dashed curves. Curves 1 correspond to the case of finite barriers while 2 - to infinitely high barriers. It is seen from this figure that at small wire di- 


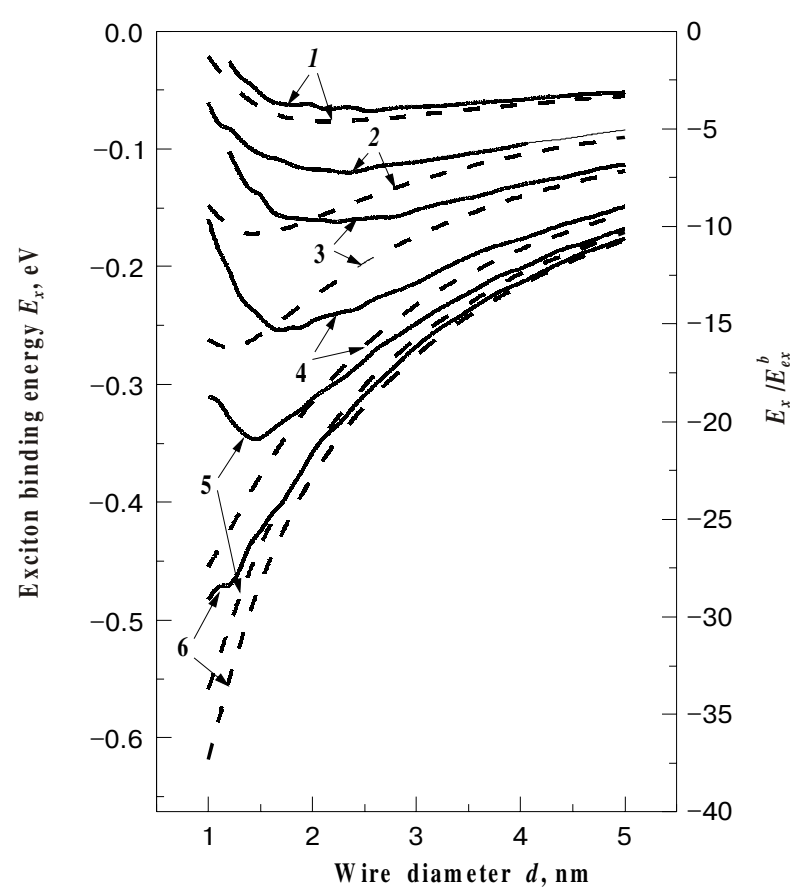

Fig. 3. Exciton binding energies in $\mathrm{Si}_{-} \mathrm{SiO}_{x}$ quantum wires as functions of QW diameter. Solid curves are obtained by variational method while dashed by perturbation matrix method. Curves: $1-x=0.25,2-x=0.75,3-x=1.00,4-x=1.50,5-$ $x=1.75,6-x=2.00$.

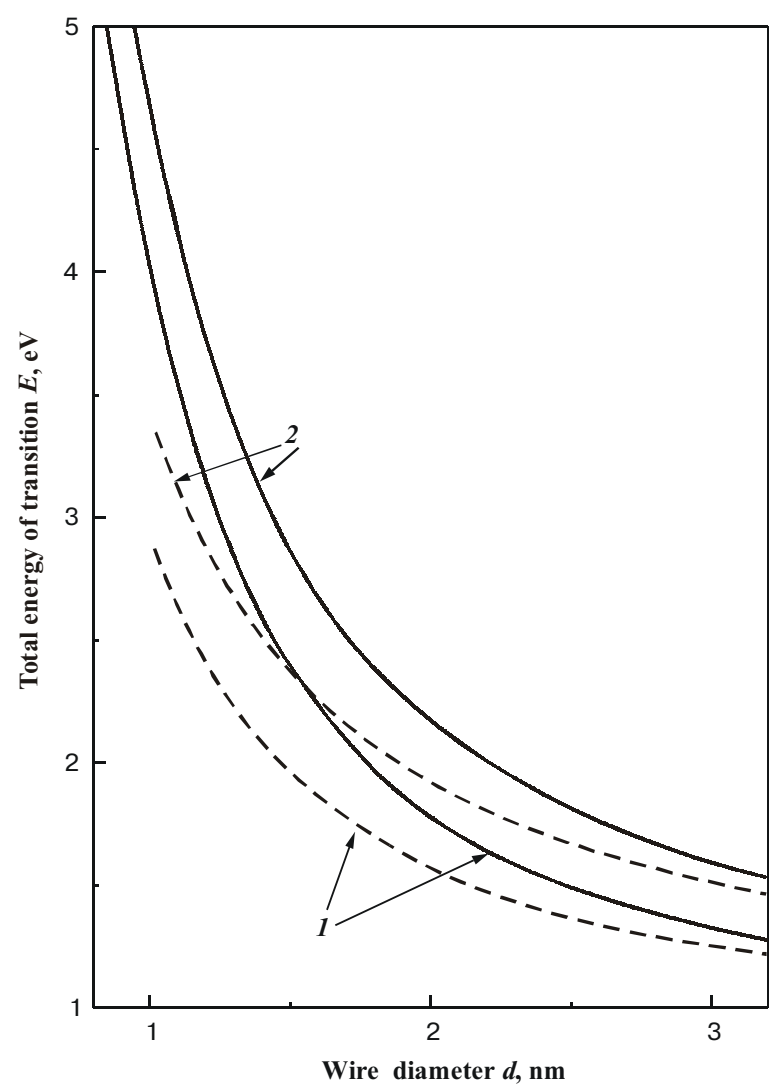

Fig. 4. Band-gap energy in $\mathrm{Si}_{-} \mathrm{SiO}_{2} \mathrm{QW}$ (solid curves) and the energy of the ground state exciton transition (dashed curves) as functions of wire thickness. Curves $l$ correspond to realistic finite band off-sets while curves 2 to infinitely large band off-sets.

SQO, 6(2), 2003 ameters effective band gap in finite barrier QW is considerably narrower than in the idealized case of infinitely high barriers. It is seen also that electron-hole bounding into exciton state leads to a substantial additional lowering of the energy of radiative transitions in QW.

\section{Radiative recombination rate of the excitons in QW}

The radiative recombination rate have been calculated using standard scheme. Initial state in QW system is characterized by empty electromagnetic field states and occupied exciton state with energy $E$ of internal motion, wave vector $\mathbf{K}$ of exciton center-of-mass motion and polarization $\sigma$, while final - by empty exciton states and one photon state characterized by the energy $\hbar \omega$, wavevector $\boldsymbol{\eta}$ and polarization $\lambda$. The probability of transition between these states per unit time in accordance with "golden rule" is expressed by the formula $W_{\mathbf{K}, \mathfrak{\eta}, \omega, \lambda, \sigma}=$ $=(2 \pi / \hbar)\left|V_{\mathbf{K}, \boldsymbol{\eta}, \omega}, \lambda, \sigma\right|^{2} \delta\left(E+\hbar^{2} K^{2} / 2 M_{e x}-\hbar \omega\right)$, where $V_{\mathbf{K}, \mathfrak{\eta}, \omega, \lambda, \sigma}$ is the matrix element of the exciton-photon interaction operator, $M_{e x}=\bar{m}_{e}+\bar{m}_{h}$ is the average translational exciton mass. Single-particle approximation of multi-electron problem is used in the following. We assumed also that only two bands contribute to radiative recombination process. The initial state wave function of electron subsystem, when all electrons occupy valence band states, can be written in a form of antisymmetrized product of valence band Wannier functions [13], $\Psi_{0}\left(\mathbf{r}_{1}, \mathbf{r}_{2}, \ldots \mathbf{r}_{N}\right)=\hat{A} \prod_{i, v} a_{\mathbf{q}_{i, v}}^{V}\left(\mathbf{r}_{j}\right)$, where $\mathbf{q}_{i}$ are the coordinates of crystal sites, $\mathbf{r}_{j}$ are the coordinates of electrons (spin variables are omitted for simplicity), total number of sites $N_{q}=N, \hat{A}$ is the antisymmetrization operator, $a_{\mathbf{q}_{i}, v}^{V}$ are the valence band Wannier functions localized at a site $\mathbf{q}_{i}$ and characterized by polarization index $n=1,2,3$. For the case of one electron excited into conduction band state the electron subsystem wave function in exciton representation [13] can be written as $\Psi_{\mathbf{K}, \sigma}\left(\mathbf{r}_{1}, \mathbf{r}_{2}, \ldots \mathbf{r}_{N}\right)=\sum_{\beta} \widetilde{F}(\boldsymbol{\beta}) \Phi_{\mathbf{K}, \boldsymbol{\beta}}^{\sigma}\left(\mathbf{r}_{1}, \mathbf{r}_{2}, \ldots \mathbf{r}_{N}\right)$, where $\boldsymbol{\beta}$ is the distance between sites at which electron and hole are localized,

$$
\Phi_{\mathbf{K}, \boldsymbol{\beta}}^{\sigma}=N^{-1 / 2} \times \sum_{\mathbf{R}} \exp (i \mathbf{K R}) \mathrm{X}_{\mathbf{R}, \mathbf{R}+\boldsymbol{\beta}}^{\sigma}\left(\mathbf{r}_{1}, \mathbf{r}_{2}, \ldots, \mathbf{r}_{N}\right),
$$

wave function $X_{\mathbf{R}, \mathbf{R}+\boldsymbol{\beta}}^{\sigma}$ corresponds to the state with an electron excited from the $\sigma$-polarized valence Wannier state at a site $\mathbf{R}$ into conduction band Wannier state at a site $\mathbf{R}+\boldsymbol{\beta}$,

$$
\mathrm{X}_{\mathbf{R}, \mathbf{R}+\boldsymbol{\beta}}^{\sigma}\left(\mathbf{r}_{1}, \mathbf{r}_{2}, \ldots, \mathbf{r}_{N}\right)=\hat{A} \prod_{i, V} a_{\mathbf{q}_{i} \neq \mathbf{R}, v \neq \sigma}^{V}\left(\mathbf{r}_{j}\right) a_{\mathbf{R}+\boldsymbol{\beta}}^{C}\left(\mathbf{r}_{j^{\prime} \neq j}\right) .
$$

For the ground exciton state in $\mathrm{QW}$, when longitudinal and transversal motions of carriers are completely separated, $\Psi_{\mathbf{K}, \sigma}$ takes the following form:

$$
\begin{aligned}
& \Psi_{K_{\|}, \mathrm{K}_{0 \mathrm{x}}, \sigma}=\sum_{\beta_{\|}} \tilde{F}\left(\beta_{\|}\right) N_{\|}^{-1 / 2} \sum_{R_{\|}} \exp \left(i K_{\|} R_{\|}\right) \times \\
& \times \sum_{\mathbf{s}} \exp \left(i \mathbf{K}_{0 x} \mathbf{s}\right) \tilde{\chi}_{e}(\mathbf{s}) \sum_{\mathbf{t}} \tilde{\chi}_{h}(\mathbf{t}) \times
\end{aligned}
$$




\section{D.V. Korbutyak et al.: Characteristics of confined exciton states ...}

$\times \hat{A} \prod_{i, v} a_{\mathbf{q}_{i} \neq \mathbf{R}_{\|}+\mathbf{t}, v \neq \sigma}^{V}\left(\mathbf{r}_{j}\right) a_{\mathbf{R}_{\|}+\boldsymbol{\beta}_{\|}+\mathbf{s}}^{C}\left(\mathbf{r}_{j^{\prime} \neq j}\right)$,

where $\boldsymbol{s}$ and $\boldsymbol{t}$ are the transversal coordinates of sites, $\mathbf{R}_{\|}$ are the crystal sites coordinates along $\mathrm{QW}, \boldsymbol{\beta}_{\|}$is the distance between electron and hole along QW,$N_{\|}$is the total number of sites along QW. Within such approach $\widetilde{F}, \tilde{\chi}_{e}$ and $\tilde{\chi}_{h}$ are the envelope functions introduced in previous section, but smeared over sites, i.e. $\tilde{\chi}_{e(h)}=b \chi_{e(h)}$, $\widetilde{F}=\sqrt{b} F$, where $b$ is the site edge length.

Operator of the electron-photon interaction is expressed by a standard formula $\hat{V}=-e n /(m c) \sum_{l} \mathbf{A}\left(\mathbf{r}_{l}\right) \hat{\mathbf{p}}_{l}$, where $\mathbf{A}(\mathbf{r})$ is the vector-potential of electromagnetic field at a point $\mathbf{r}$ and $\hat{\mathbf{p}}_{l}=-i \hbar \nabla_{l}$ is the momentum operator of the $l$-th electron. For a quantized electromagnetic field the vector-potential is expressed as $\mathbf{A}\left(\mathbf{r}_{l}\right)=\sqrt{2 \pi \hbar /(V \omega)} \times$ $\times(c / n) \mathbf{e}_{\lambda} \exp \left(-i \eta \mathbf{r}_{l}\right)$, where $V$ is the system volume, $\omega$ is the photon frequency, $\boldsymbol{\eta}=(\omega n / c) \mathbf{e}_{h}$ is the photon wave vector, $\mathbf{e}_{\lambda}$ is the unit polarization vector, $n$ is the refraction index.

Taking into account that Wannier functions are strongly localized at corresponding crystal sites the ma-

trix element $V_{\mathbf{K}, \boldsymbol{\eta}, \omega, \lambda, \sigma}=<\Psi_{0}\left|\hat{V}_{\boldsymbol{\eta}, \omega, \lambda}\right| \Psi_{\mathbf{K}_{\|}, K_{0 x}, \sigma}>$ after integration over all electron variables and replacement of the remaining sum by the integral reduces to the following form:

$V_{\mathbf{K}, \boldsymbol{\eta}, \omega, \lambda, \sigma}=-\sqrt{\frac{2 \pi \hbar L \omega}{V}} F(0) \delta_{\mathbf{K}_{\|}, \boldsymbol{\eta}_{\|}} M_{\perp}\left\langle C\left|\mathbf{e}_{\lambda} \hat{\mathbf{d}}\right| V, \sigma>\right.$,

where $\mathrm{L}$ is the QW length, $\hat{\mathbf{d}}=$ er is the electric dipole operator. Transversal component of the matrix element is

expressed as $M_{\perp}=\int \exp \left[i\left(\mathbf{K}_{0 x}-\boldsymbol{\eta}_{\perp}\right) \boldsymbol{\rho}\right] \chi_{e}(\rho) \chi_{h}(\rho) d^{2} \boldsymbol{\rho}$.

Due to negligibly small transversal photon wave vectors $\boldsymbol{\eta}_{\perp}$ comparing to the $\mathbf{K}_{0 x}$ value the last formula can be written as

$M_{\perp} \approx 2 \pi \int_{0}^{\infty} J_{0}\left(K_{0 x} \rho\right) \chi_{e}(\rho) \chi_{h}(\rho) \rho d \rho$.

If total probability of QW exciton radiative recombination is a subject of interest we have to summarize contributions from all states in the exciton 1D-subband and integrate the result over all possible final photon states accounting for all conservation laws:

$$
\begin{aligned}
& W_{\text {total }}^{Q W}=\frac{V}{(2 \pi)^{2}} \int d \eta_{\|} \int \eta_{\perp} d \eta_{\perp} \int d \omega \delta\left(\omega-\frac{c}{n} \sqrt{\eta_{\|}^{2}+\eta_{\perp}^{2}}\right) \times \\
& \times \sum_{\lambda} \sum_{\sigma} \sum_{\mathbf{K}_{\|}} \frac{2 \pi}{\hbar}\left|V_{\mathbf{K}_{\|}, \boldsymbol{\eta}_{\|}, \omega, \lambda, \sigma}\right|^{2} \delta\left(E+\frac{\hbar^{2} \eta_{\|}^{2}}{2 M_{e x}}-\hbar \omega\right) f(\hbar \omega),
\end{aligned}
$$

where $f$ is the exciton distribution function, integration over $\omega$ is introduced to account for photon dispersion low. With $2 / 3$ weight coefficient accounting for the fraction of degenerate valleys from which radiative transitions can occur in QW and using Boltzmann distribution for the excitons in 2D-subband the final result for the probability of exciton radiative recombination per unit time is expressed by the following formula:

$W_{r}^{Q W}=\frac{16}{9} \frac{p^{2}}{\hbar} F^{2}(0)\left(\frac{2 \pi \hbar^{2}}{M_{e x} k_{B} T}\right)^{1 / 2}\left(\frac{n E}{\hbar c}\right)^{3} M_{\perp}^{2}$,

where $p^{2}$ is the square of the matrix element $\left\langle\mathrm{C}\left|\mathbf{d}_{\lambda}\right| \mathrm{V}\right\rangle$, $F(z)$ is the wave function of mutual electron-hole longitudinal motion (see previous section), $E$ is the exciton recombination energy, $T$ is the temperature. At large wire diameters the matrix element $M_{\perp}$ turns to zero (only phonon or impurity assisted transitions contribute to the exciton luminescence in bulk silicon). However, in thin QWs the probability of exciton luminescence without phonon (or impurity) assistance increases substantially due to increased overlapping of electron and hole wave functions of transversal motion. An excess pulse $\sim \hbar K_{0}$ in this case is transferred directly to the QW as a whole.

Fig. 5 demonstrates the calculated dependencies of the reduced exciton radiative lifetime $\tau_{x r}$ on QW diameter. Calculations were made using value $\tau_{x}^{b r}=10^{-3} \mathrm{~s}$ of background (LO-phonon or impurity assisted) radiative recombination time of excitons reported in the literature for high-quality silicon material and the value $K_{0 x}=0.85 \cdot(2 \pi / a)$ for the X-valley distance from the center of Brillouin zone (silicon lattice constant $a=0.54 \mathrm{~nm}$ ). The reduced exciton radiative lifetime $\tau_{x r}$ is determined by the reciprocal law relation $1 / \tau_{x r}=$ $=1 / \tau_{x}^{b r}+W_{r}^{Q W}$. Oscillating character of the dependencies $\tau_{x r}(d)$ and $M_{\perp}(d)$ is associated with the interference between $X$-electron and $\Gamma$-hole wave functions in the region of their transversal localization (oscillations should be absent in the case of direct band gap materials). The

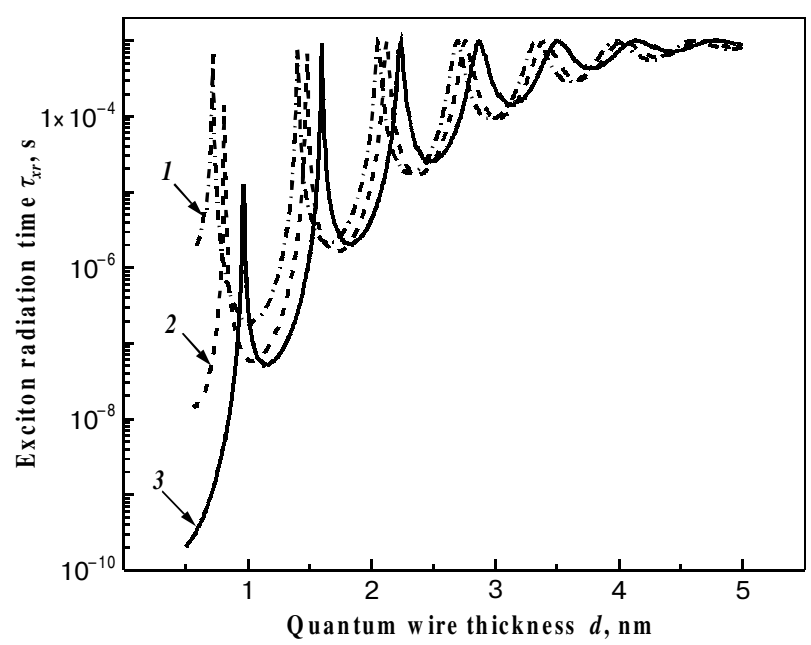

Fig. 5. Exciton radiative lifetime $\tau_{x r}$ as function of $\mathrm{Si}_{-}-\mathrm{SiO}_{\mathrm{x}} \mathrm{QW}$ thickness. Curves: $1-x=1.5,2-x=1.75,3-x=2$. 


\section{D.V. Korbutyak et al.: Characteristics of confined exciton states ...}

interference effect becomes most pronounced at QW thicknesses $d$ comparable with $1 / K_{0 x}$ value.

\section{Quantum efficiency and intensity of the exciton PL in QW structures}

We consider here the exciton luminescence which results from electron-hole $(e-h)$ pairs generation by laser irradiation with photon energy exceeding QW band gap but lower than band gap of barrier material. Excited electrons and holes relax quickly to the lowest $\mathrm{X}$ - and $\Gamma$-valley states and due to the Coulomb interaction have chance to create Wannier-Mott excitons considered in previous sections. The exciton states under certain conditions can serve as an important channel for the radiative recombination processes.

A theoretical analysis of the exciton luminescence in semiconductors at high temperatures was made in [14]. It was shown that the exciton mechanism of edge luminescence could play an important role manifesting itself at least in comparable intensities of exciton and interband radiative transitions. Note that at quasi equilibrium conditions the relationship between exciton concentration $n_{x}$ and concentration of free electron-hole pairs $n_{e-h}$ is governed by the parameter $E_{x} / k_{B} T$ where $E_{x}$ is the absolute value of the exciton binding energy in the ground state. As $E_{x} / k_{B} T$ grows (e.g., due to quantum confinement and dielectric enhancement effects in QW systems) the ratio $n_{x} / n_{e-h}$ increases as well. This leads to an enhancement of the excitonic component of the recombination processes. The relationship between exciton and electron (hole) concentrations at quasi-equilibrium takes up the following form:

$$
n_{x}=n p / n^{*} \text {, }
$$

where $n_{x}, n$ and $p$ are the total non-equilibrium 1D concentrations (particles per unit QW length) of excitons, electrons and holes, respectively,

$$
n^{*}=N \exp \left(-\frac{E_{x}}{k_{B} T}\right) \text {. }
$$

Here $N=\sqrt{\mu k_{B} T /\left(2 \pi \hbar^{2}\right)}, \mu$ is the reduced exciton mass. Expressions (21) and (22) can be obtained from the equality of quasi-chemical potentials of the exciton and electron-hole subsystems under quasi equilibrium conditions. Taking into account that at intense homogeneous laser excitation the concentrations of excess carriers are equal, $\Delta p=\Delta n$, and that total concentrations are determined practically by the excess carriers $(p \sim \Delta p, n \sim \Delta n)$ the conservation laws for the fluxes of $e-h$ pairs and excitons reduce to the following forms:

$$
\begin{aligned}
& \frac{d p}{d t}=-\frac{p}{\tau_{p}}-\gamma\left[n p-n^{*} n_{x}\right]+I_{0} \alpha d c, \\
& \frac{d n_{x}}{d t}=-\frac{n_{x}}{\tau_{x}}+\gamma\left[n p-n^{*} n_{x}\right], \\
& S Q O, 6(2), 2003
\end{aligned}
$$

where $d$ is the QW diameter, $c=k d(k \sim 1)$ is the formfactor, $\tau_{p}$ and $\tau_{x}$ are the lifetimes of $e-h$ pairs and excitons, respectively, $\gamma$ is the probability of electron-hole association into exciton in $\mathrm{QW}, p$ is in fact the concentration of excess electron-hole pairs. Eqs. (23) are valid only for the case of uniform excitation. We also suppose that the times of electron (hole) relaxation in the conduction (valence) bands are much smaller than $\tau_{p}$ and $\tau_{x}$.

Under steady-state conditions the integral intensity of the exciton luminescence in QW is expressed as $J=n_{x} /\left(\tau_{x r} c\right)$, while the internal quantum efficiency as $\eta=J /\left(\alpha d I_{0}\right)$. For a concentration of $e-h$ pairs substantially exceeding the concentration of equilibrium carriers, the following formula for $\eta$ can be obtained:

$$
\begin{aligned}
& \eta=\left(2 \frac{\tau_{x}}{\tau_{x r}}\right)^{2}\left[\sqrt{\left(\frac{\tau_{x}}{\tau_{p}}\right)^{2}\left(\frac{n^{*}}{\tau_{x r} I_{0} \alpha c d}\right)+4 \frac{\tau_{x}}{\tau_{x r}}}+\right. \\
& \left.\frac{\tau_{x}}{\tau_{p}} \sqrt{\frac{n^{*}}{\tau_{x r} I_{0} \alpha c d}}\right]^{-2} .
\end{aligned}
$$

In Fig. 6 the dependencies of internal quantum efficiency of the exciton luminescence on QW thickness are shown. They have been obtained using following parameters: nonradiative recombination exciton lifetime $\tau_{x}^{n r}=10^{-6} \mathrm{~s}$, background radiative recombination lifetime of excitons $\tau_{x}^{b r}=10^{-3}$ s, carriers recombination life time $\tau_{p}=10^{-5} \mathrm{~s}$, absorption coefficient $\alpha=10^{4} \mathrm{~cm}^{-1}$, intensity of the exciting light $I_{0}=10^{18} \mathrm{~cm}^{-2} \mathrm{~s}^{-1}$, temperature $T=300 \mathrm{~K}$. The total reduced exciton lifetime $\tau_{x}$ is determined by reciprocal law relation $1 / \tau_{x}=1 / \tau_{x r}^{n r}+$ $+1 / \tau_{x}^{b r}+W_{r}^{Q W}$, where $W_{r}^{Q W}$ is expressed by (20) ${ }^{x}$.

Values of lifetimes $\tau_{x}^{n r}$ and $\tau_{x}^{b r}$ used in our calculations are those reported for bulk silicon. In general, they can be functions of nanostructure thickness too (like $\left.W_{r}^{Q W}\right)$. Background lifetime $\tau_{x}^{b r}$ for exciton radiative recombination with phonon participation seems to rise in NSs compared to its bulk value due to phonon spectrum quantization and corresponding decrease of exciton scattering. With this lifetime fixed we underestimate the quantum efficiency, but really in the case of $\mathrm{Si}_{-} \mathrm{SiO}_{\mathrm{x}}$ nanostructures it should not be an important factor due to expected large $\tau_{x}^{b r}$ values in comparison with substantially decreased exciton radiative recombination lifetimes. Situation with nonradiative recombination rates is more complex due to existence of different channels for nonradiative recombination. The rate of nonradiative multi-phonon recombination seems to decrease with NS thickness for the already outlined reason, but even more important in this case is a relative decrease in NSs of a number of bulk centers of recombination (in extremely narrow NSs with $d \rightarrow 0$ such centers can be absent at all). However, instead of bulk centers a new "surface" channel of nonradiative recombination should come into force. The last channel can be suppressed by compensa- 


\section{D.V. Korbutyak et al.: Characteristics of confined exciton states ...}
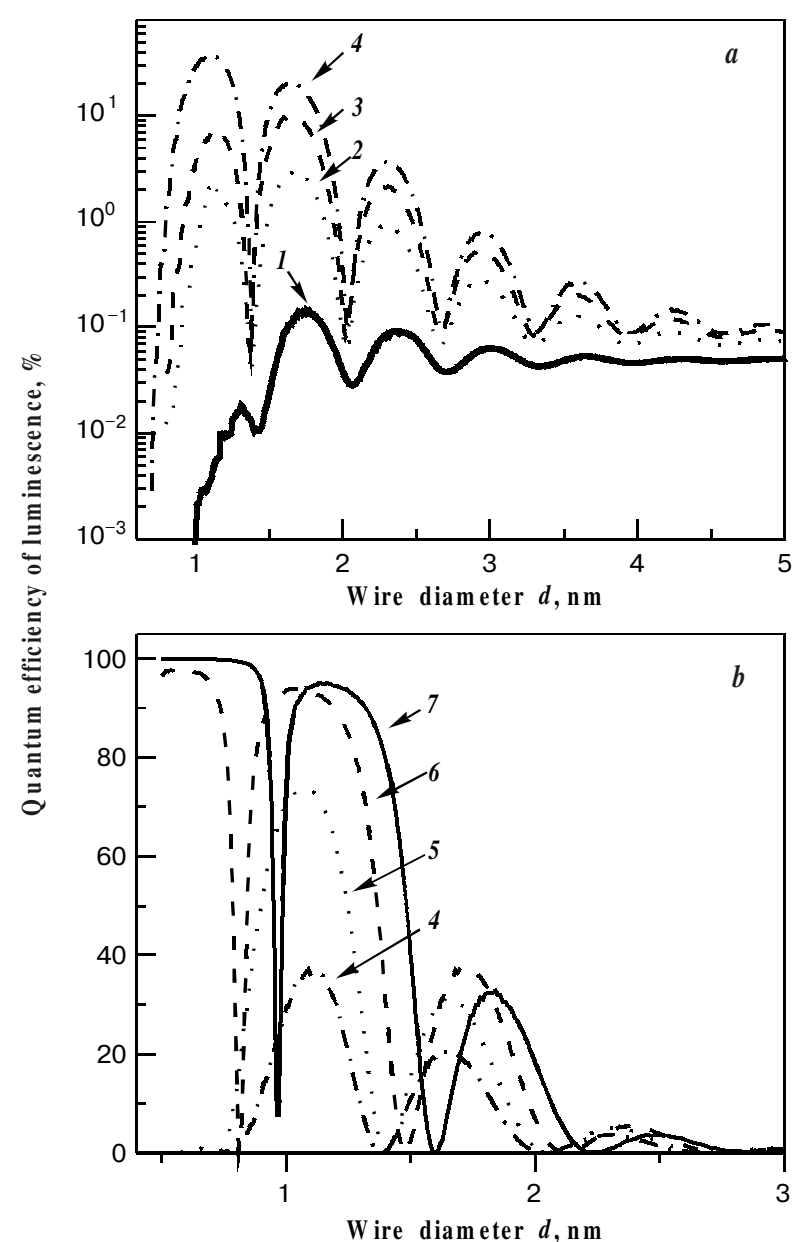

Fig. 6. Thickness dependencies of the internal quantum efficiency of the exciton luminescence in $\mathrm{Si}_{-} \mathrm{SiO}_{x}$ quantum wires. Intensity of laser excitation $I_{0}=10^{18} \mathrm{~cm}^{-2} \mathrm{~s}^{-1}$. Curves: $I-$ $x=0.25,2-x=0.75,3-x=1.00,4-x=1.25,5-x=1.50$, $6-x=1.75,7-x=2.00$.

tion of dangling bonds at the interface. In the case of silicon it can be done by hydrogen or oxigen passivation. One more channel contributing to the nonradiative recombination dependence on NS width is associated with the Auger process, but it becomes important at higher intensities of laser excitation.

The above speculations justify to some extent the approximation of structure-independent rates of background and nonradiative recombination. With all recombination rates being structure-dependent the exciton luminescence characteristics, of course, will deviate from those calculated in the present work, but due to the fact that radiative recombination rates in indirect gap NSs with $d<3 \mathrm{~nm}$ approach those of direct gap materials the changes most likely will reduce to a smoothening of peculiarities in the calculated dependencies.

An oscillating character of these dependencies is dictated by oscillations in the matrix element (18) due to the interference of the electron and hole wave functions within region of their transversal localization. The overall increase of quantum efficiency with QW thickness decrease occurs due to lifting a restriction on electron transitions from $X$-valley states of conduction band into $\Gamma$-valley states of valence band. Such radiative transitions become possible due to a substantial increase of the probability of exciton pulse transfer directly to quantum structure as a whole at sufficiently small thicknesses $d(<3 \mathrm{~nm})$. In the case of $\mathrm{Si}_{-} \mathrm{SiO}_{\mathrm{x}}$ quantum wires with composition parameter lower than $x \sim 1$ quantum efficiency achieves maximal values at $d \sim 1.5-1.7 \mathrm{~nm}$ and then falls at smaller $d$. This effect is directly associated with a decrease in effective barrier height.

In Fig. 7 thickness dependencies of the integral intensity of exciton luminescence in QWs are shown for the intensity of laser excitation $I_{0}=10^{18} \mathrm{~cm}^{-2} \mathrm{~s}^{-1}$. All notations in this figure are the same as in Fig. 6. In comparison with quantum efficiency dependencies an additional shift of maximum luminescence intensities to the region of larger QW diameters is observed.

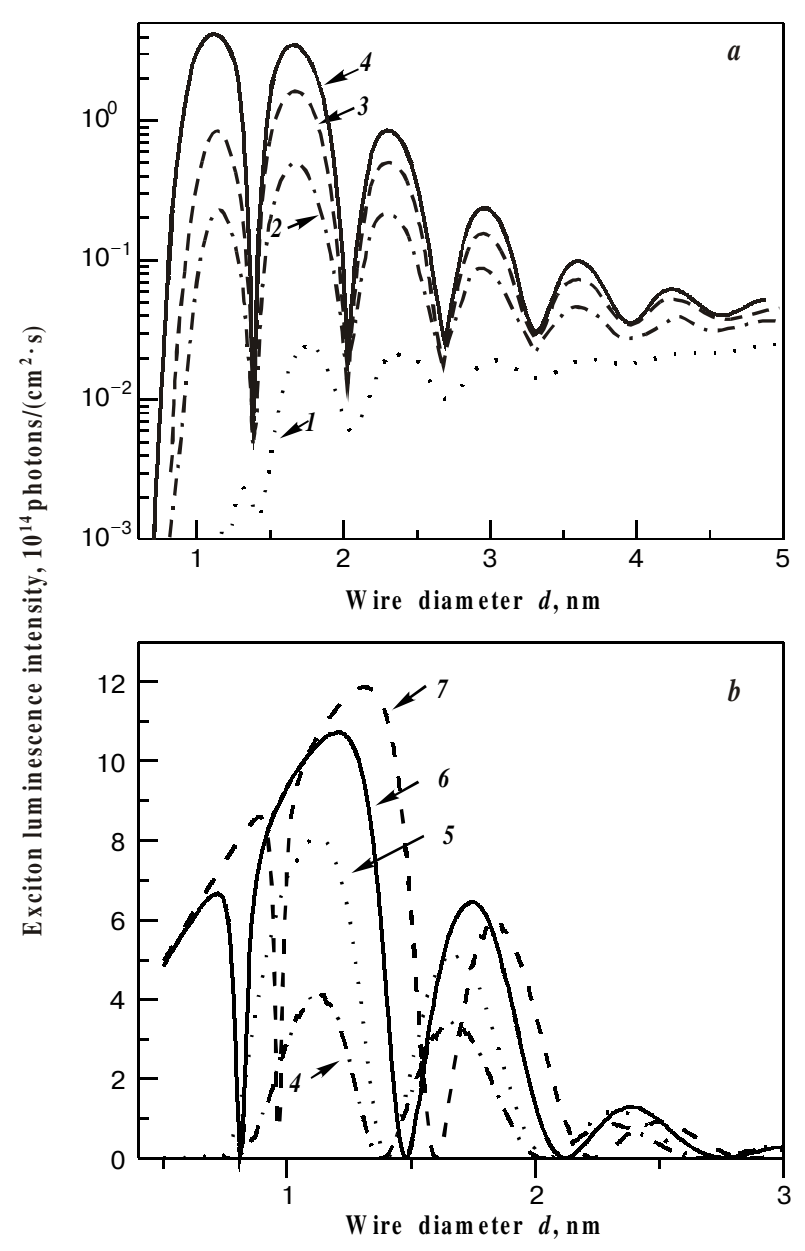

Fig. 7. Thickness dependencies of the integral intensity of the exciton luminescence in $\mathrm{Si}_{-} \mathrm{SiO}_{x}$ quantum wires. Intensity of laser excitation $I_{0}=10^{18} \mathrm{~cm}^{-2} \mathrm{~s}^{-1}$. Curves: $l-x=0.25,2-x=0.75$, $3-x=1.00,4-x=1.25,5-x=1.50,6-x=1.75,7-x=2.00$. 


\section{D.V. Korbutyak et al.: Characteristics of confined exciton states ...}

Finally, it is of interest to calculate the exciton PL spectra and to compare them with the experimental data for porous silicon. In real systems contributions from QWs with different thicknesses $d$ should be taken into account. Distribution of QWs in porous silicon can be described by the function $\partial F(d)=1 /(\sigma d \sqrt{2 \pi}) \exp \left[-\left(\ln d-\ln d_{0}\right)^{2} / 2 \sigma^{2}\right] \partial d$ [15]. To account for possible apparatus function influence and scattering effects (by lattice vibrations, interface imperfections, etc.) the energy conserving delta function in (19) has been replaced by Lorenz function $\Gamma /\left(2 \pi\left[(E-\hbar \omega)^{2}+\Gamma^{2} / 4\right]\right)$. Converting calculated $E(d, x)$ dependencies for $\mathrm{Si}_{-} \mathrm{SiO}_{\mathrm{x}} \mathrm{QW}$ into $d(E, x)$ dependencies and averaging final result over possible parameters $x$ in the oxidized layers around QWs in porous silicon the spectral density of the exciton PL per unit QW can be expressed as

$$
\begin{aligned}
& \frac{\partial I}{\partial \omega}=\frac{\hbar}{\sigma_{x} \sigma(2 \pi)^{2}} \int \partial x \int J(E, x) \frac{\partial \ln d(E, x)}{\partial E} \times \\
& \times \exp \left[-\frac{\left(\ln d(E, x)-\ln d_{0}\right)^{2}}{2 \sigma^{2}}\right] \times \\
& \times \exp \left[-\frac{\left(x-x_{0}\right)^{2}}{2 \sigma_{x}^{2}}\right] \frac{\Gamma}{(\hbar \omega-E)^{2}+\Gamma^{2} / 4} \partial E .
\end{aligned}
$$

In Fig. 8 the calculated PL spectra are compared with experimental ones [16] measured in different samples of porous silicon. From the fitting of PL spectra the distribution of quantum wires over their thickness and oxidization parameter $x$ can be found (e.g., thickness distri-

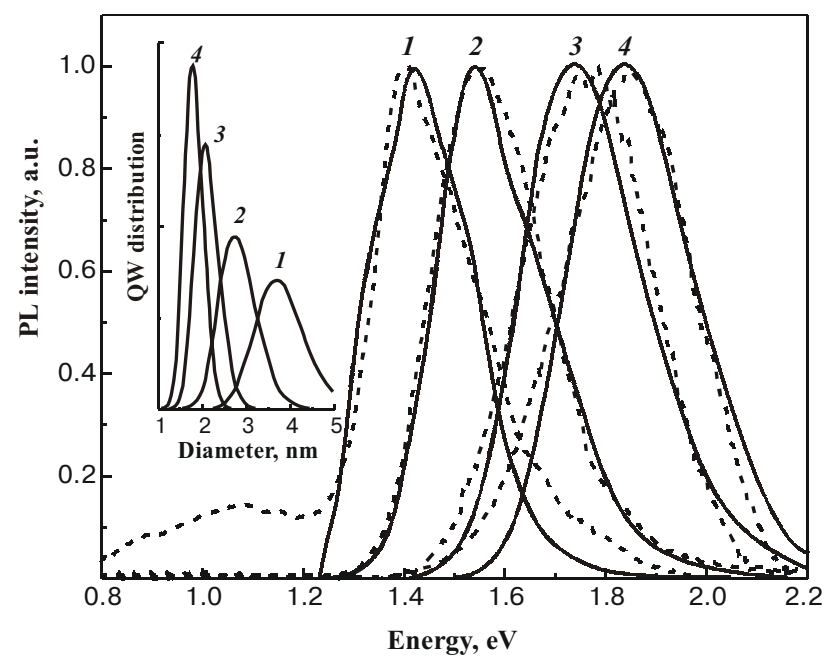

Fig. 8. Calculated (solid) and experimental ([16], dashed) PL spectra of porous silicon. Corresponding QW thickness distributions are shown in the insert. Fitting parameters for the curves: $1-\left(d_{0}=3.7 \mathrm{~nm}, \sigma=1.16, x_{0}=2, \sigma_{x}=0.25\right) ; 2-\left(d_{0}=2.8 \mathrm{~nm}, \sigma=\right.$ $\left.=1.16, x_{0}=1.65, \sigma_{x}=0.25\right) ; 3-\left(d_{0}=2.1 \mathrm{~nm}, \sigma=1.14, x_{0}=1.58\right.$, $\left.\sigma_{x}=0.2\right) ; 4-\left(d_{0}=1.8 \mathrm{~nm}, \sigma=1.12, x_{0}=1.44, \sigma_{x}=0.1\right)$.

$S Q O, 6(2), 2003$ butions corresponding to different PL spectra are shown in the insert). Calculating all PL spectra we used the fixed value $\sim 25 \mathrm{meV}$ of the damping parameter $\Gamma$ (this value corresponds approximately to the room-temperature value of $k T$ ). Other parameters of fitting are given in figure.

\section{Conclusions}

It has been shown for $\mathrm{Si}_{-} \mathrm{SiO}_{\mathrm{x}}$ quantum wires that in the case of high barriers realized at $x \sim 2$ the intensity of exciton luminescence rises substantially with NS thickness decrease. In NS with $d \sim 2 \mathrm{~nm}$ or smaller the internal quantum efficiency of exciton luminescence can achieve almost $100 \%$ even at room temperatures. For lower barrier heights the situation is more complex. For $x<1$ the quantum efficiency of the exciton luminescence achieves maximal values in QWs with $d \sim 1.5 \mathrm{~nm}$ and drops in narrower QWs. It is shown that confinement and dielectric enhancement effects in NSs with finite barriers are responsible for large exciton binding energies although these energies are lower than in the case of infinitely high barriers. An analogous effect of finite barriers is demonstrated for the total energy of exciton transition in QW structures. It is shown, that experimental PL spectra of porous silicon can be sufficiently well described considering an ensemble of quantum wires with different thickness and oxidization parameters of the adjacent $\mathrm{SiO}_{\mathrm{x}}$ layers. Using our approach, $\mathrm{QW}$ distribution functions can be determined from the fitting between experimental and theoretical spectra.

\section{Acknowledgements}

This work was supported by INTAS grant (INTAS Call 2001 NANO-0444), Russian-Ukrainian Program "Nanophysics and nanoelectronics" and State Fundamental Researches Foundation of Ukraine.

\section{References}

1. L.V. Keldysh, Coulomb interaction in thin films of semiconductors and semimetals // Pisma Journ. Exp and Theor. Phys. 29(11), pp. 716-719 (1997) (in Russian).

2. V.S. Babichenko, L.V. Keldysh, A.P. Silin, Coulomb interaction in thin semiconductor and semimetal wires // Fizika Tverdogo Tela 22(4), pp. 1238-1240 (1980) (in Russian).

3. E.A. Muljarov, S.G. Tikhodeev, Dielectric enhancement of excitons in semiconductor quantum wires // Journ. Exp and Theor. Phys. 111(1), pp. 274-282 (1997) (in Russian).

4. E. Shangina, Electron scattering phenomena in GaAs single quantum wire. Theory and calculations // Phys. Low-Dim. Struct. 11/12, pp.171-182 (2000).

5. X. Leyronas, M. Combescot, Quantum wells, wires and dots with finite barrier: analytical expressions for the bound states // Sol. St. Commun. 119, pp.631-635 (2001).

6. J.D. Jackson, Classical Electrodynamics, Eds. John Wiley \& Sons Inc., New York - London (1962).

7. R. Loudon, One-dimensional hydrogen atom // American Journal of Physics 27(9), pp. 649-655 (1959). 


\section{D.V. Korbutyak et al.: Characteristics of confined exciton states ...}

8. M.G. Lisachenko, E.A. Konstantinova, P.K. Kashkarov, V.Yu. Timoshenko, Dielectric effect in silicon quantum wires // Phys. Stat. Solidi (a) 182 (1), pp. 297-300 (2000).

9. E.A. Muljarov, S.G. Tikhodeev, N.A. Gippius, T. Ishishara, Excitons in self-organized semiconductor-insulator superlattices // Phys. Rev. B51 (20), pp. 14370-14378 (1995).

10. E. Holzenkämpfer, F.M. Richter, J. Stuk, U. Voget-grote, // J. Non-Cryst. Solids 32 , pp. 327- (1979).

11. G. Zuther, Dielectric and optical properties of $\mathrm{SiO}_{\mathrm{x}} / /$ Phys. Stat. Sol. (a) 59 (1), pp. K109-K113 (1980).

12. V.G. Litovchenko, V.G. Popov, A.A. Evtukch, B.N. Romanjuk, Characteristics of the insulator-semiconductor systems with multi-layer and graded-bandgap insulators
/ Preprint of the Institute of Semiconductors,76-76, Kiev, 1991.

13. R. Knox, Theory of Excitons, Academic Press, New York London (1963).

14. A.V. Sachenko, Yu.V. Kryuchenko, Excitonic effects in bandedge luminescence of semiconductors at room temperatures // Semiconductor Physics, Quantum Electronics \& Optoelectronics 3 (2), pp. 150-156 (2000)

15. Y. Kanemitsu, Light emission from porous silicon and related materials // Physical Reports 263 , pp.1-91 (1995)

16. G. Polisski, O.M. Sreseli, A.V. Andrianov, F. Koch, Infrared luminescence of porous silicon at room temperature // Fizika i tekhnika poluprovodnikov 31 (3), pp.365-369 (1997). 